\title{
$\checkmark$ Hemorrhagic Acquired Pial Arteriovenous \\ Case \\ Report Fistula Occurring after Mechanical Thrombectomy: A Case Report
}

Koichiro Shindo, Tatsuya Ogino, Tomoki Fuchizaki, Hideki Endo, Yohei Maruga, Toshiichi Watanabe, Kenji Kamiyama, Toshiaki Osato, and Hirohiko Nakamura

Objective: We report a patient in whom thrombectomy for occlusion of the middle cerebral artery was performed, and a pial arteriovenous fistula (AVF) developed in the relevant vascular area.

Case Presentation: The patient was a 72-year-old male. In 2011, thrombectomy with a Penumbra system for right M1 occlusion causing right cerebral infarction was performed. Recanalization was achieved (modified thrombolysis in cerebral infarction $[\mathrm{TICl}] 2 \mathrm{~b}$ ) although occlusion of the M2 anterior trunk remained. After 1 week, MRA confirmed complete recanalization. The course was favorable, and he was discharged. After 5 years, convulsion occurred, and he was brought to our hospital by ambulance. MRI showed subcortical hemorrhage of the right precentral gyrus. DSA confirmed an arteriovenous (AV) shunt between the right central artery and vein of Trolard, which had not been present. There was no nidus, leading to a diagnosis of a pial AVF. Under craniotomy, the shunt point was disconnected.

Conclusion: Intraoperative findings suggested the involvement of cortical vein thrombosis after thrombectomy in the etiology.

Keywords pial arteriovenous fistula, adult, mechanical thrombectomy

\section{Introduction}

A pial arteriovenous fistula (AVF) is a relatively rare vascular lesion. Previously, it had been classified as a subtype of arteriovenous malformation (AVM), ${ }^{1)}$ but it has gradually been recognized independently because there is no nidus between the artery and vein. The pathogenesis remains to be clarified, but hemorrhage initially occurs in many adult patients. ${ }^{2)}$ In such patients, conservative treatment leads to a poor prognosis; ;) aggressive treatment may be necessary. In this study, we report a patient in whom mechanical thrombectomy with Penumbra system (Penumbra Inc., Alameda, CA, USA) for occlusion of the right middle cerebral artery

Department of Neurosurgery, Nakamura Memorial Hospital, Sapporo, Hokkaido, Japan

Received: April 8, 2018; Accepted: July 31, 2018

Corresponding author: Koichiro Shindo. Department of Neurosurgery, Nakamura Memorial Hospital, South 1, West 14, Chuo-ku, Sapporo, Hokkaido 060-8570, Japan

Email: k.shindo04hmrfc@gmail.com

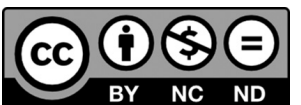

This work is licensed under a Creative Commons Attribution-NonCommercialNoDerivatives International License.

C2019 The Japanese Society for Neuroendovascular Therapy was performed, and an acquired pial AVF developed in the relevant vascular area, and review the literature.

\section{Case Presentation}

Case: A 72-year-old male.

Medical history: Paroxysmal atrial fibrillation, cerebral infarction.

Present illness: In 2011, intravenous thrombolysis with recombinant tissue plasminogen activator (rt-PA) and thrombectomy with Penumbra system were performed to treat right cerebral infarction and occlusion of the right middle cerebral artery (M1 occlusion) (Fig. 1a). Aspiration was conducted using an aspiration pump, and a Penumbra 4MAX reperfusion catheter was inserted to the site of occlusion while adopting a separator so that thrombi were collected. Recanalization (modified thrombolysis in cerebral infarction [TICI] 2b) was achieved although occlusion of the M2 anterior trunk remained (Fig. 1b). Right internal carotid angiography at the time of recanalization showed vein of Trolard perfusion descending from the superior sagittal sinus (SSS) to the superficial middle cerebral vein (SMCV) (Fig. 1c). MRA 1 week after thrombectomy confirmed recanalization of the M2 anterior trunk. 

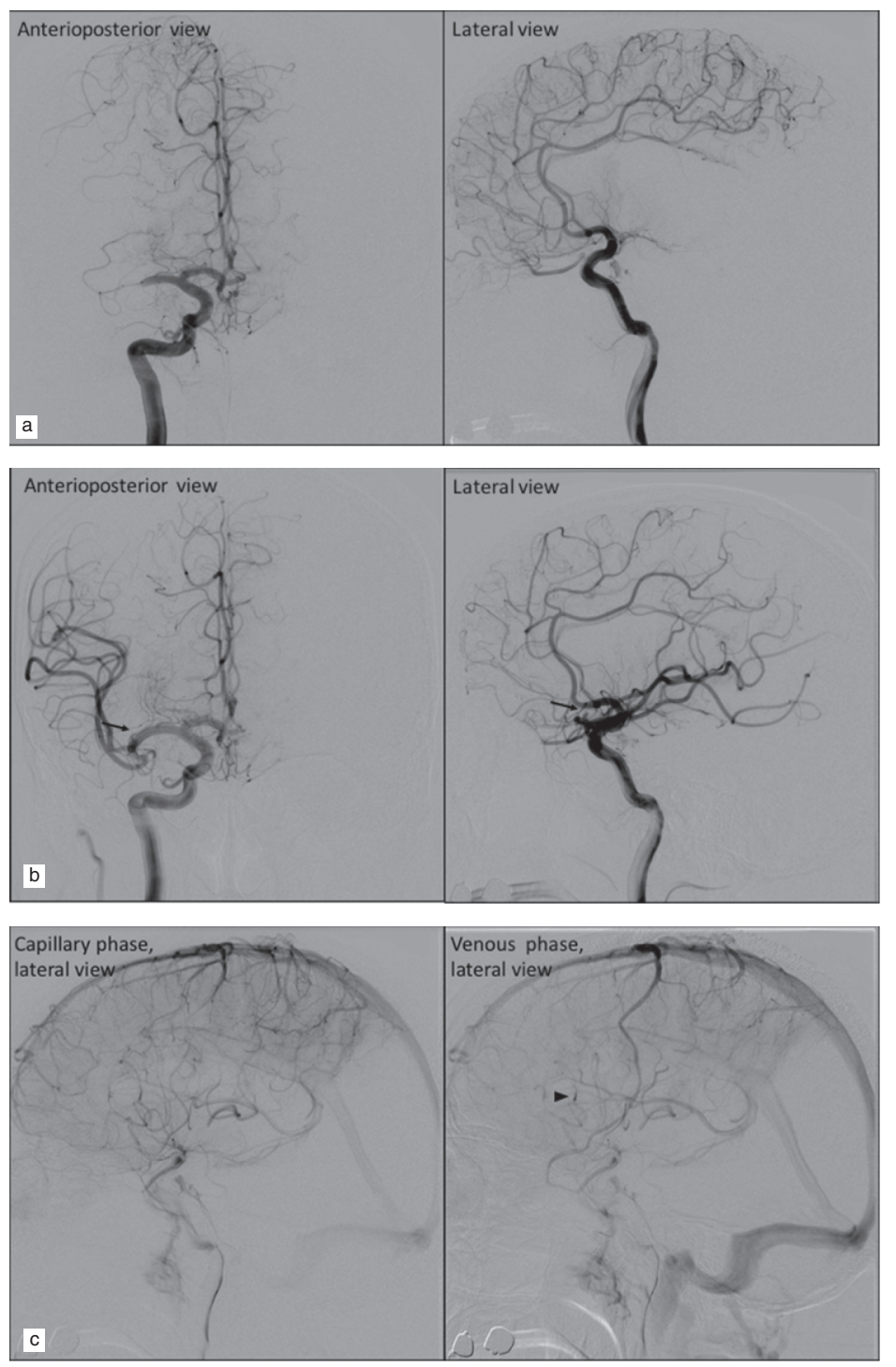

Fig. 1 (a) Right internal carotid angiography showed occlusion at the M1 segment of the middle cerebral artery. (b) Penumbra reperfusion catheter was advanced to the occlusion site and thrombus was removed. Right internal carotid angiography showing the right M2 anterior trunk occlusion (arrow) after multiple pass. ( $\mathrm{TICl} 2 \mathrm{~b}$ ). (c) Right internal carotid angiography of post thrombectomy. Due to a residual thrombus, contrast medium stagnating at the right M2 anterior trunk (arrowhead). The flow of precentral vein was descending from SSS to SMCV. SMCV: superficial middle cerebral vein; SSS: superior sagittal sinus; TICl: thrombolysis in cerebral infarction

The postoperative course was favorable, and the patient was discharged through 1-month admission, with a modified Rankin Scale (mRS) score of 1. At this point, atrial fibrillation was not detected, and Warfarin was selected as a secondary prophylactic agent. Follow-up was continued at the outpatient clinic. After 5 years, convulsion occurred, and the patient was brought to our hospital by ambulance. Brain MRI and CT revealed subcortical hemorrhage of the right frontal lobe (precentral gyrus) (Fig. 2a), and he was admitted. DSA suggested an arteriovenous (AV) shunt between the right central artery and vein of Trolard, which was not observed at the time of thrombectomy in 2011. Although the site of hemorrhage was the precentral gyrus, symptoms were mild. On DSA after 2 months, the AV shunt was more clearly visualized, and there was no nidus between the artery and vein, leading to a diagnosis of a pial AVF (Fig. 2b). To prevent additional hemorrhage, shuntpoint disconnection by craniotomy was scheduled. Before surgery, Warfarin was discontinued, and heparin replacement was conducted. 

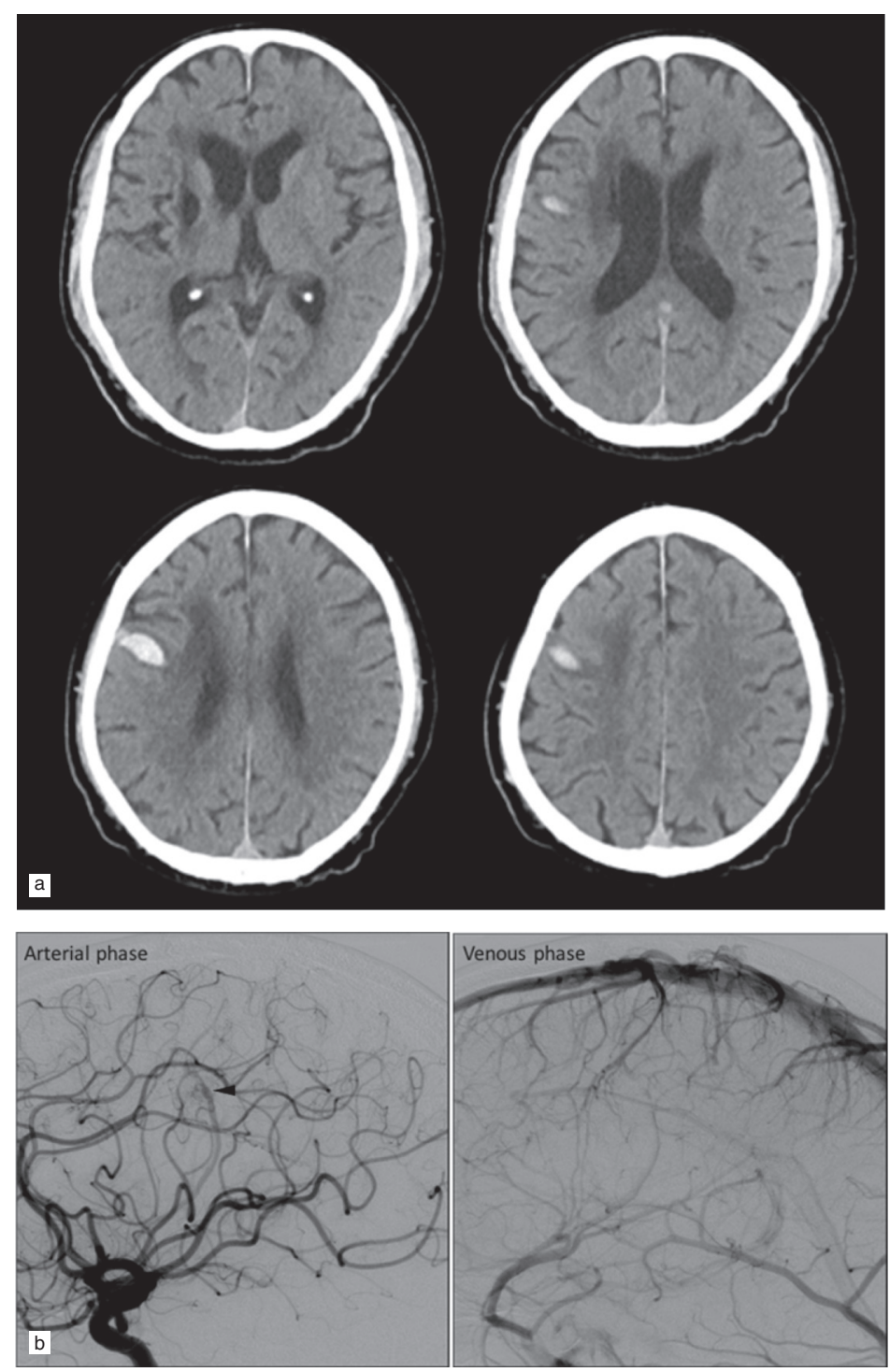

Fig. 2 (a) Axial plain CT showing subcortical hemorrhage in the right precentral gyrus. (b) Right internal carotid angiography showing a pial AVF with the feeding artery arising from the central artery. A pial AVF drains to the vein of Trolard (arrowhead). AVF: arteriovenous fistula

Intraoperative findings (Fig. 3a): Craniotomy involving the shunt point was performed. On incising the dura mater, arachnoid thickening and cloudiness at the shunt site were observed. Hemosiderin deposition was noted, suggesting the presence of old subarachnoid hemorrhage. The arachnoid around the AV shunt was exfoliated, and the vascular structure was denuded. In the vein of Trolard, mixed venous blood and AV-shunt arterialized blood was macroscopically observed. The AV shunt was present in a cortical vein joining a precentral vein (vein of Trolard), and the affected vein had formed a venous pouch. Arterioles connected to the venous pouch from central and precentral arteries as feeding arteries. The affected vein extended into the deep area of the central sulcus, and there was no nidus. As diagnosed before surgery, a pial AVF was suggested. The AV shunt involving the affected cortical vein was separated from the peripheral tissue. The intraoperative findings included venous thrombosis at the deep area of the affected cortical vein (Fig. 3b). In the process of affected cortical vein dissection and removal, the old-subcorticalhemorrhage-related hematoma cavity was reached, supporting that the pial AVF was an etiological factor for cerebral hemorrhage. Indocyanine green (ICG) fluorescence angiography and intraoperative cerebral angiography confirmed the disappearance of the AV shunt, and surgery was completed. 


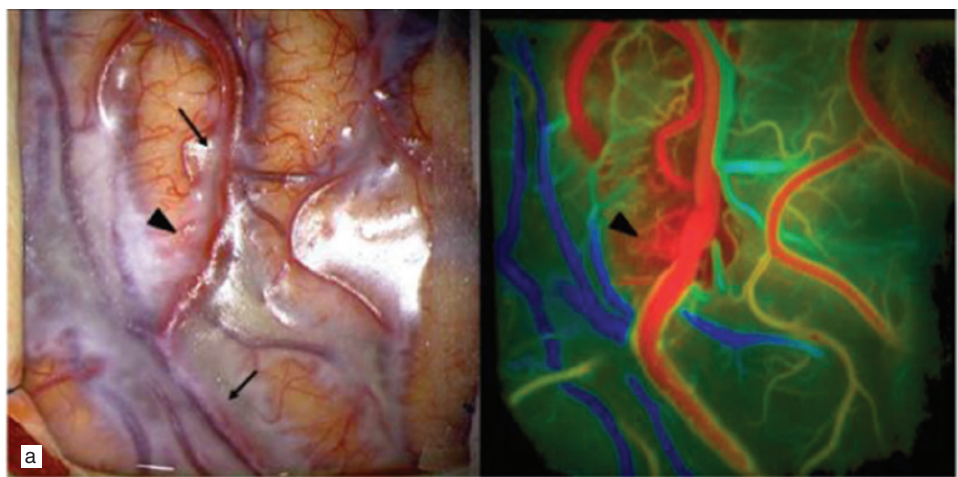

Intraoperative view
Color map during ICG injection

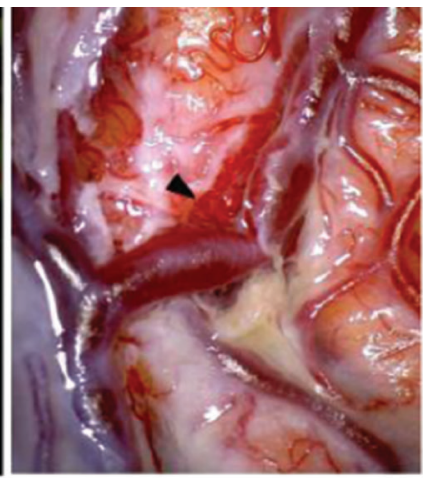

Intraoperative view after removal arachnoid membrane

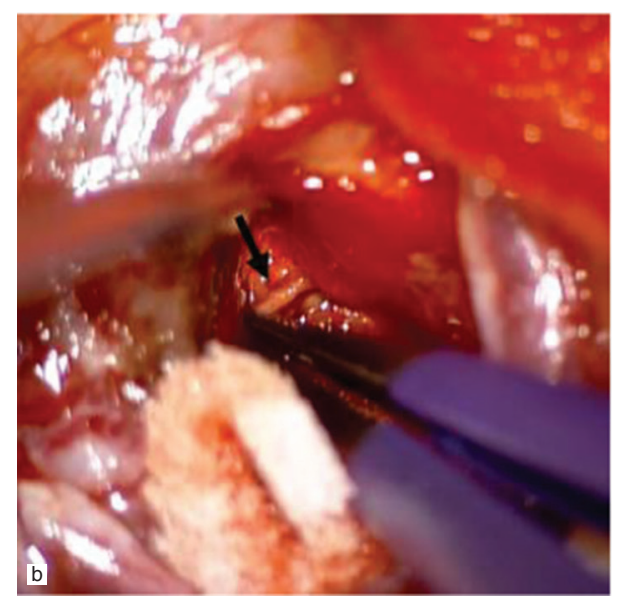

Fig. 3 (a) Intraoperative photograph of the precentral vein (the vein of Trolard) appearing red (arrows) and there is a venous pouch in the side of precentral gyrus. Feeding arteries connecting to the venous pouch (arrowhead). (b) Intraoperative photograph. The vessel (arrow) in the deep portion of the central sulcus being recognized as thrombosed vein. ICG: indocyanine green

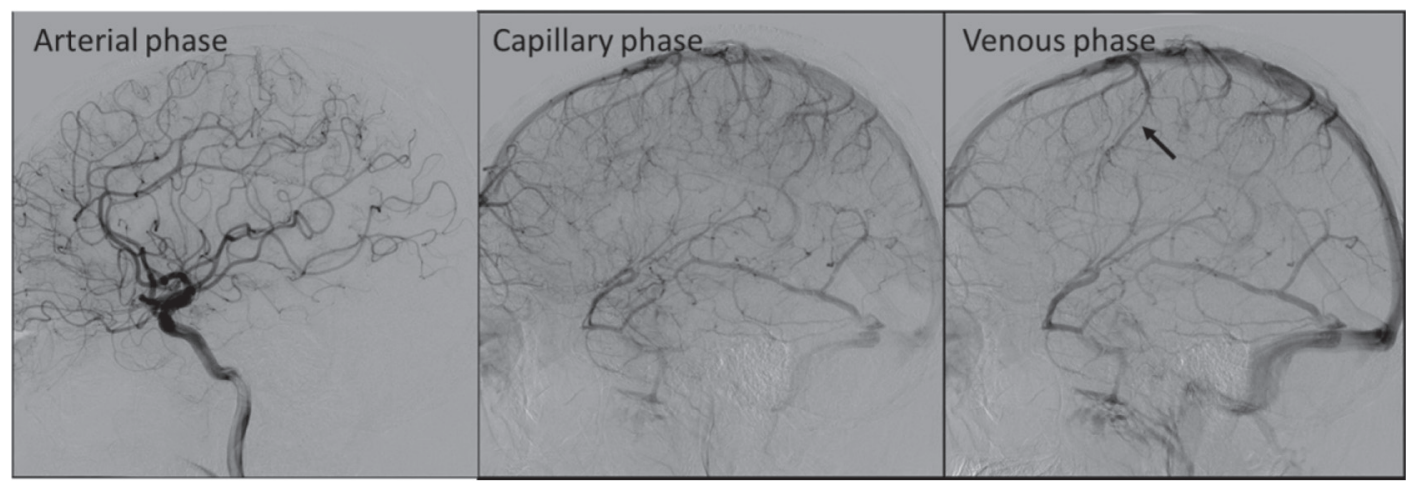

Fig. 4 Postoperative right carotid angiogram showing complete obliteration of the fistula. The flow of the precentral vein is ascending to SSS (arrow). SSS: superior sagittal sinus

Postoperative DSA: The AV shunt had disappeared, and the perfusion direction of the precentral vein had changed to an ascending one (SSS direction), involving the shunt site as a border. The SMCV remained descending (Fig. 4).
As paroxysmal atrial fibrillation was detected on preoperative electrocardiography, the regimen was switched to a direct oral anticoagulant (DOAC) after confirming the absence of hemorrhagic complications. The postoperative 

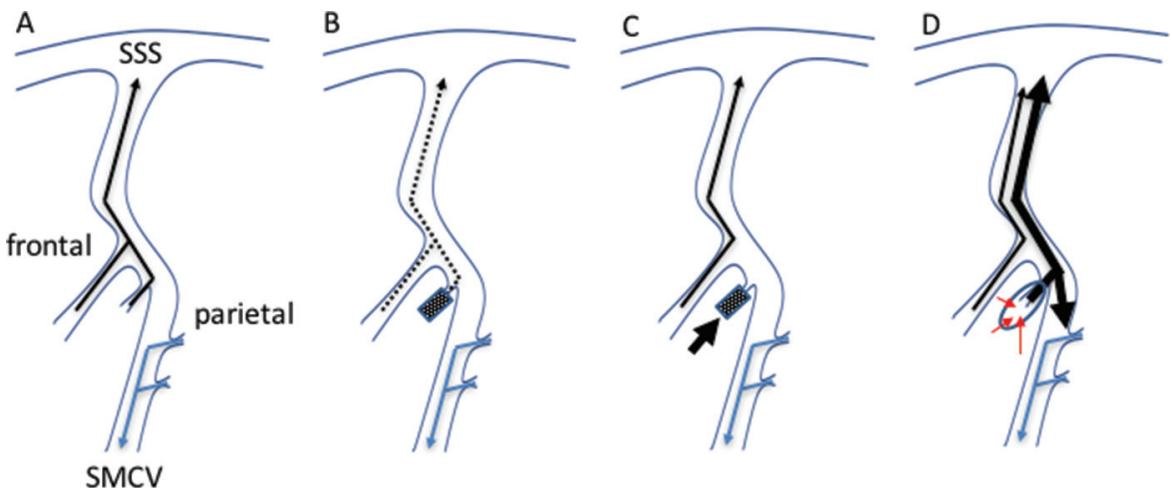

Fig. 5 Schematic drawing explaining a possible mechanism for pial AVF development. (A) Normal venous perfusion. The cortical veins from the frontal lobe perfused to SSS and those from the parietal lobe to SMCV. (B) During M2 anterior trunk occlusion, venous perfusion pressure from the frontal lobe was lower and the cortical vein seemed to be thrombosed. (C) After recanalization of the M2 anterior trunk, venous perfusion pressure from the frontal lobe was recovered and venous pressure of the occluded vein locally increased. (D) At the time of the bleeding in the precentral gyrus, the pial AVF was indicated with drainage to the cortical vein connecting to the vein of Trolard. AVF: arteriovenous fistula; SMCV: superficial middle cerebral vein; SSS: superior sagittal sinus

course was favorable, and the patient was discharged, with mRS score of 1 .

\section{Discussion}

A pial AVF is an intracranial AV shunt disease involving a single or several pial arteries as feeders and a single cortical vein as a drainer, which differs from AVM with a nidus. Its incidence accounts for $1.6 \%-4.7 \%$ of intracranial AV shunt diseases; ${ }^{4,5)}$ this disease is relatively rare. Pial AVFs are classified into two types: congenital and acquired AVFs. In children, it was reported that congenital pial AVFs were associated with hereditary diseases, such as hereditary hemorrhagic telangiectasia (HHT) and capillary malformation (CM)-AVM. ${ }^{6,7)}$ The pathogenesis of acquired AVFs may involve transient venous hypertension following head trauma, ${ }^{8)}$ surgery or sinus thrombosis. ${ }^{9)}$ Etiological factors for onset include mass effects, hemorrhage, convulsion, and heart failure. Yang et al. reported that convulsionrelated AVF onset was frequent in children aged $\leq 15$ years, and that hemorrhage-related AVF onset was frequent in adults aged $\geq 16$ years. $^{2)}$

According to a study, ${ }^{10)}$ an improvement was achieved by anticoagulant therapy alone in a patient with a nonhemorrhagic pial AVF complicated by sinus thrombosis. However, another study indicated that the mortality rate after conservative treatment was $63 \%$ in patients with hemorrhage. ${ }^{3)}$ Yang et al. reported that varices with arterialpressure-buffering effects were absent in most adult patients, suggesting that hemorrhage-related AVF onset was frequent. ${ }^{2}$ In adult- or hemorrhage-onset patients, radical treatment is considered to be necessary in the early phase.

There is no nidus, differing from AVM; therefore, removal of the lesion site is not always necessary, and shunt-point disconnection alone may be sufficient. ${ }^{2)}$ To treat pial AVFs, direct surgery, endovascular treatment, or a combination of the two procedures is selected. For superficial lesions, either treatment may be possible, but endovascular treatment may be advantageous for deep or eloquent-area lesions, which are difficult to treat by direct surgery. According to several studies, ${ }^{11-14)}$ embolic materials used for endovascular treatment include balloons, coils, glue, polyalcohol, and Onyx. However, if the influx of an embolic material into a draining vein without complete shunt-point disconnection affecting venous outflow occurs, delayed hemorrhage may occur. Furthermore, extracerebral distal embolism must also be considered. According to a review published by Yang et al.,2) the obliteration rates achieved by direct surgery and endovascular treatment were $96.8 \%$ and $86.5 \%$, respectively; direct surgery may be more advantageous with respect to radical cure. However, treatment methods should be selected based on detailed preoperative examination of lesion localization or difficulties in approaching the shunt point.

Matsubara et al. reported that capillaries were generated in the nidus on pathological examination of thrombotic AVM, suggesting that recanalization or reperfusion in the presence of venous thrombosis induces angiogenesis, contributing to the formation of an AV shunt.9) 
Case reports of pial AVFs related to sinus thrombosis have been published, but, to our knowledge, no study has reported their appearance after mechanical thrombectomy. Matsubara et al. presented patients with dural/pial AVFs during follow-up of SSS thrombosis, ${ }^{9}$ ) and indicated that the pial AVF had developed at the site of occluded-corticalvein recanalization. In the present case, MRA 1 week after thrombectomy confirmed recanalization of the M2 anterior trunk. The cortical vein (draining vein) may have been occluded a few days before arterial recanalization. As an in vivo response to the occluded cortical vein, angiogenesis may have been induced, and arterial recanalization may have increased the cortical vein pressure in the region, leading to the formation of a pial AVF (Fig. 5). Recently, acute-phase recanalization therapy has been increasingly performed, and pial AVFs should be considered a complication that may occur in an occluded-blood-vesseldominated area. Much attention must be paid to fine changes on periodic examinations, such as MRI/MRA.

\section{Conclusion}

We reported a patient with a pial AVF in whom hemorrhage occurred 5 years after mechanical thrombectomy. Regional venous hypertension related to changes in venous perfusion after thrombectomy may have been involved in the pathogenesis.

\section{Disclosure Statement}

There is no conflict of interest for the first author and coauthors.

\section{References}

1) Yasargil M. Microneurosurgery IIIA: AVM of the brain, history, embryology, pathological considerations, hemodynamics, diagnostic studies, microsurgical anatomy. Stuttgart: Georg Thieme Verlag KG, 1987.
2) Yang WH, Lu MS, Cheng YK, et al: Pial arteriovenous fistula: a review of literature. Br J Neurosurg 2011; 25: 580-585.

3) Nelson PK, Niimi Y, Lasjaunias P, et al: Endovascular embolization of congenital intracranial pial arterio-venous fistulas. Neuroimaging Clin N Am 1992; 2: 309-317.

4) Lv X, Li Y, Jiang C, et al: Endovascular treatment of brain arteriovenous fistulas. AJNR Am J Neuroradiol 2009; 30: 851-856.

5) Lv X, Jiang C, Li Y, et al: Clinical outcomes of endovascular treatment for intracranial pial arteriovenous fistulas. World Neurosurg 2010; 73: 385-390.

6) Kikuchi K, Kowada M, Sasajima H: Vascular malformations of the brain in hereditary hemorrhagic telangiectasia (Rendu-Osler-Weber disease). Surg Neurol 1994; 41: 374-380.

7) Ishiguro $\mathrm{T}$, Komiyama $\mathrm{M}$, Terada $\mathrm{A}$, et al: [Pial arteriovenous fistulas]. Niche Neuro-Angiology Conference 2011. http://nnac.umin.jp/nnac/di5huipuroguramu_files/16\%20 Ishiguro.pdf (Accessed May 21, 2011) (in Japanese)

8) Nomura S, Ishikawa O, Tanaka K, et al: Pial arteriovenous fistula caused by trauma: a case report. Neurol Med Chir (Tokyo) 2015; 55: 856-858.

9) Matsubara S, Satoh K, Satomi J, et al: Acquired pial and dural arteriovenous fistulae following superior sagittal sinus thrombosis in patients with protein S deficiency: a report of two cases. Neurol Med Chir (Tokyo) 2014; 54: 245-252.

10) Kadotani M, Wajima D: [Pial arteriovenous fistula with sinus thrombosis treated by anticoagulation therapy]. Currently Practical Neurosurgery 2016; 26: 68-73. (in Japanese)

11) Viñuela F, Drake CG, Fox AJ, et al: Giant intracranial varices secondary to high-flow arteriovenous fistulae. J Neurosurg 1987; 66: 198-203.

12) Viñuela F, Fox AJ, Kan S, et al: Balloon occlusion of a spontaneous fistula of the posterior inferior cerebellar artery. Case report. J Neurosurg 1983; 58: 287-290.

13) Wang YC, Wong HF, Yeh YS: Intracranial pial arteriovenous fistulas with single-vein drainage. Report of three cases and review of the literature. J Neurosurg 2004; 100: 201-205.

14) Kim HM, Cho JH, Kim KH: Onyx embolization of intracranial pial arteriovenous fistula. J Cerebrovasc Endovasc Neurosurg 2016; 18: 291-295. 